\title{
Reduced expression of endogenous secretory receptor for advanced glycation endproducts in hippocampal neurons of Alzheimer's disease brains*
}

\author{
Ichiro Nozaki1,2, Takuo Watanabe1, Makoto Kawaguchi ${ }^{3}$, Hiroyasu Akatsu ${ }^{4}$, Koichi \\ Tsuneyama $^{5}$, Yasuhiko Yamamoto' ${ }^{1}$ Kazuyo Ohe ${ }^{1}$, Hideto Yonekura ${ }^{6}$, Masahito Yamada², \\ and Hiroshi Yamamoto ${ }^{1}$
}

${ }^{1}$ Department of Biochemistry and Molecular Vascular Biology, and ${ }^{2}$ Department of Neurology and Neurobiology of Aging, Kanazawa University Graduate School of Medical Science, Kanazawa; ${ }^{3}$ Department of Pathology, Niigata Rousai Hospital, Joetsu; ${ }^{4}$ Choju Medical Institute, Fukushimura Hospital, Toyohashi, ${ }^{5}$ First Department of Pathology, School of Medicine, University of Toyama, Toyama; and ${ }^{6}$ Department of Biochemistry, Kanazawa Medical University, School of Medicine, Uchinada, Japan

Summary. The receptor for advanced glycation end-
products (RAGE) is a cell-surface multiligand receptor,
which interacts with amyloid $\beta(A \beta)$, a key protein in
Alzheimer's disease (AD). RAGE-A $\beta$ interaction is thought
to be associated with pathological progression in AD. A
splice variant of RAGE, endogenous secretory RAGE
(esRAGE) can act as a decoy receptor for RAGE ligands
that would prevent the progression of some pathologic
conditions. In this study, the expression of esRAGE

Received December 13, 2007

* This work was supported in part by grants-in-aid from the Japan Society for the Promotion of Sciences (nos. 18590260 and 19390085); grants (C2006-4 and C2007-1) for Collaborative Research from Kanazawa Medical University and a grant (H2007-10) for Project Research from the HighTechnology Center of Kanazawa Medical University (to H. Yonekura).

Address for correspondence: Takuo Watanabe, M.D., Ph.D., Associate Professor, Department of Biochemistry and Molecular Vascular Biology, Kanazawa University Graduate School of Medical Science, 13-1 Takara-machi, Kanazawa, 920-8640, Japan

Te1.: +81-76-265-2181, Fax: +81-76-234-4217

E-mail: takuo@med.kanazawa-u.ac.jp in the hippocampal tissues from AD brains compared with control (non-AD) was examined by immunohistochemistry and Western blot analysis. Semiquantitative immunohistochemical analysis of hippocampal tissues using esRAGE-specific antibody revealed significantly decreased immunoreactivities in pyramidal cells in CA1 and CA3 regions of AD compared with non-AD. On the other hand, immunoreactivities of astrocytes for esRAGE significantly increased in those regions. Dentate granule cells and astrocytes showed essentially invariant immunoreactivities between $A D$ and non-AD. Changes in esRAGE immunoreactivity in CA3 neurons and astrocytes were observed from the early pathological stages. Moreover, the esRAGE-immunoreactive bands of AD samples were weaker than those of non-AD samples in Western blot analysis. The results indicate that low expression of esRAGE in the hippocampus would be associated with the development of $A D$.

\section{Introduction}

The receptor for advanced glycation endproducts (RAGE) is a member of the immunoglobulin superfamily of cell surface receptors (Schmidt et al., 1992; Yonekura et al., 2005). Human RAGE has a 322-amino acid extracellular portion which is composed of one $\mathrm{V}$-type immunoglobulin domain and two C-type immunoglobulin domains, a single 19-amino acid hydrophobic transmembrane-spanning 
domain and a 41-amino acid, highly-charged cytoplasmic tail: V-domain is critical for ligand binding, and the cytoplasmic domain is essential for intracellular signaling (Huttunen et al., 1999). RAGE is a multiligand receptor and endogenous ligands other than advanced glycation endproducts (AGE), such as amphoterin/high mobility group box 1 (HMGB-1) (Hori et al., 1995), S100/ calgranulin (Hofmann et al., 1999), transthyretin (Sousa et al., 2000), and amyloid $\beta$ (A $\beta$ ) (Yan et al., 1996; Mackic et al., 1998) have been identified. The RAGEsignaling cascade is not yet fully understood, but ligand binding to RAGE has been reported to induce cellular oxidant stress to activate the transcription factor nuclear factor- $\kappa \mathrm{B}(\mathrm{NF}-\kappa \mathrm{B})$ through a cascade of intracellular signaling that involves mitogen-activated protein kinase activation (Lander et al., 1997; Stern et al., 2002).

$\mathrm{AD}$ is a progressive neurodegenerative disease with amyloid burden (i.e. senile plaques), tau pathology (i.e. neurofibrillary tangles), and chronic inflammation. RAGE is also a receptor for $\mathrm{A} \beta$, the major component of senile plaques, and RAGE-A $\beta$ interaction is considered to be associated with neurotoxicity in AD brain. The neural dysfunction mediated by RAGE would be caused by the following mechanisms: induction of neuronal oxidative stress (Yan et al., 1996); activated microglial inflammatory responses (Lue et al., 2001); and increased $\mathrm{A} \beta$ influx into the brain across the blood-brain barrier (Deane et al., 2003).

We recently identified a naturally occurring secreted soluble form of RAGE in human and in mouse RAGE, and this is expressed in multiple organs including the central nervous system (Yonekura et al., 2003; Cheng et al., 2005; Harashima et al., 2006). This variant is generated by alternative splicing, and is called endogenous secretory RAGE (esRAGE); esRAGE is composed of the extracellular domain and unique C-terminal sequence that lacks both the transmembrane and intracellular domains, and has been shown to act as a decoy receptor that inhibits RAGE signaling (Yonekura et al., 2003). It is thought that esRAGE suppresses the progression of diseases or pathological conditions by the inhibition of signaling via membrane-type RAGE (Yonekura et al., 2005). Plasma esRAGE levels are significantly decreased compared with the control group in some diseases such as atherosclerosis and diabetic retinopathy (Koyama et al., 2005; Sakurai et $a l ., 2006)$. Low levels of circulating esRAGE would cause vulnerability to those diseases. We hypothesized that low expression of esRAGE in the hippocampal neurons could be associated with susceptibility to AD in which hippocampal degeneration would be most critical. In the present study, this hypothesis was studied for human AD brains compared with control non-AD samples by using immunohistochemical methods and Western blot analysis that uses esRAGE-specific and membrane-type RAGEspecific antibodies.

\section{Materials and Methods}

\section{Subjects and tissues}

Included in this study were 19 patients with $\mathrm{AD}$ (6 men and 13 women, mean age: $85.3 \pm 6.4$ years), 21 patients with normal physiological aging or neurological diseases other than $\mathrm{AD}$ (called "non-AD"; 8 men and 13 women, mean age: $83.5 \pm 8.0$ years). The non- $\mathrm{AD}$ group involved cases in which AD was excluded by histopathological examination, including normal physiological aging $(n=4)$, cerebral infarction $(n=15)$, brain metastatic tumor $(n=1)$, and Parkinson disease $(n=1)$. Informed consent as regards participation was provided by all $\mathrm{AD}$ patients and control subjects or their legal guardians and the approval of the local ethical committee was obtained. All tissue specimens and corresponding clinical data were provided by Choju Medical Institute Brain Bank (Akatsu et al., 2002), and all examinations were performed according to the principles expressed in the Declaration of Helsinki. Their clinical characteristics are summarized in Table 1.

\section{Neuropathology}

For this study, formalin-fixed, paraffin-embedded tissue blocks of $A D$ or non-AD brains were randomly selected from autopsy specimens registered in Choju Medical Institute Brain Bank (Akatsu et al., 2002). The tissue sections were stained with hematoxylineosin and with the Gallyas-Braak method. Each brain was examined and diagnosed by two neuropathologists. The neuropathological diagnosis of AD followed the Consortium to Establish A Registry for Alzheimer's Disease (CERAD) guidelines (Mirra et al., 1991). Gallyas-Braak stains were used to determine Braak neurofibrillary scores (Braak and Braak, 1991).

\section{Antibodies}

Rabbit esRAGE-specific polyclonal antibody was raised against the unique $\mathrm{C}$-terminal 16 -amino-acid peptide (amino acids 332-347; EGFDK VREAEDSPQHM)keyhole limpet hemocyanin conjugate, and immunoaffinity-purified with an antigen peptideconjugated column as previously described (Yonekura et $a l ., 2003$ ). The specificity of the immunostaining has been proven by the absorption test (Cheng et al., 2005). 
A rabbit polyclonal antibody raised against the C-terminal intracellular domain of membrane-type RAGE (Cterminal 20 amino acids) was purchased from Santa Cruz Biotechnology, CA, USA.

\section{Immunohistochemistry and semiquantitative analyses}

Immunostaining procedures were based on a new microwave technique as described previously (Kumada et al., 2004). In brief, the section were deparaffinized, dehydrated, and treated for antigen retrieval with Target Retrieval Solution (DAKO, Carpinteria, CA, USA) in a wet chamber in a microwave oven (Type RE-11; Sharp, Tokyo; maximum $500 \mathrm{~W}$ ) for $15 \mathrm{~min}$. The sections were allowed to cool at room temperature for $30 \mathrm{~min}$, rinsed under running water for $1 \mathrm{~min}$, and then treated with $3 \%$ $\mathrm{H}_{2} \mathrm{O}_{2}$ for 5 min to exhaust endogenous peroxidase. After washing with Tris-buffered saline (TBS) containing $0.1 \%$ Tween-20 (TBS-T) and blocking with 5\% normal bovine serum albumin (BSA) (Sigma-Aldrich, St. Louis, MO, USA) for $1 \mathrm{~min}$, the sections were incubated with primary antibodies ( $5 \mu \mathrm{g} / \mathrm{m} l$ for esRAGE-specific antibody; $2 \mu \mathrm{g} /$ $\mathrm{m} l$ for membrane-type RAGE-specific antibody) in a wet chamber, with intermittent microwave irradiation (MI-77; Azumaya, Tokyo) for $10 \mathrm{~min}$. After washing with TBS$\mathrm{T}$, the peroxidase-labeled polymer for rabbit polyclonal antibody (EnVison; DAKO) was applied for $10 \mathrm{~min}$ in a wet chamber with intermittent microwave irradiation. After washing with TBS, color was developed with 3 3'- diaminobenzidine (Sigma-Aldrich). The sections were then counterstained with Meyer's hematoxylin, and cover-slipped for microscopic observation. The conditions of immunostaining and digital imaging were carefully controlled to assure the uniformity of evaluation of immunoreactivity. The intensity of immunoreactivity (color; brown) in cells at hippocampus was scored on an arbitrary scale as follows: $0=$ no staining; $1=$ weak staining; 2 = intermediate staining; and $3=$ strong staining. The proportion of cells in which the intensity of immunoreactivity is $0,1,2$, and 3 is defined as $p_{0}, p_{1}$, $\mathrm{p}_{2}$, and $\mathrm{p}_{3}$, respectively. The scoring was done by plural analysts. The immunoreactivity index (IRI) for specific cell type was calculated with the following formula: $0 \times \mathrm{p}_{0}+1 \times \mathrm{p}_{1}+2 \times \mathrm{p}_{2}+3 \times \mathrm{p}_{3}$. The following three hippocampal subdivisions were analyzed; CA1, CA3, and dentate granule cell layer. The neuron/astrocyte esRAGE index was calculated for each case using the following formula: esRAGE IRI for neurons/esRAGE IRI for astrocytes. The identification of neurons and astrocytes were based on their histological characteristics in Meyer's hematoxylin stain as follows: pyramidal cells in CA1 and CA3 have a relatively large (10-50 $\mu \mathrm{m}$ in diameter) triangularly shaped cell body and multiple dendrites; granule cells in dentate gyrus are tiny neurons that are around $10 \mu \mathrm{m}$ in diameter found in granular layer; astrocytes typically have a characteristic star-shaped cell body that is smaller than pyramidal cells, and round nuclei; oligodendrocytes that are recognizable by their small, rounded, lymphocyte-like nuclei and perinuclear halo were discriminated from astrocytes. Identification criteria for astrocytes and oligodendrocytes were confirmed by immunostaining for their specific makers, glial fibrillary acidic protein and olig2, respectively (data not shown).

\section{Western blot analysis}

Frozen blocks of hippocampal tissues were homogenized in SDS lysis buffer [62.5 mM Tris $\cdot \mathrm{HCl}(\mathrm{pH} 6.8), 2 \%(\mathrm{w} /$ v) SDS, $10 \%$ glycerol] using Polytron homogenizer (KINEMATICA, Littau-Lucerne, Switzerland) and boiled at $95^{\circ} \mathrm{C}$ for $5 \mathrm{~min}$. The lysates were centrifuged at $15,000 \mathrm{~g}$ for $5 \mathrm{~min}$ and the supernatants were

Table 1. Clinical characteristics of subjects

\begin{tabular}{|c|c|c|c|c|c|c|}
\hline Diagnosis & $\begin{array}{c}\text { Number of patients } \\
\text { (male/female) }\end{array}$ & $\begin{array}{c}\text { Age } \\
\text { (years) }\end{array}$ & $\begin{array}{c}\text { Clinical duration } \\
\text { (years) }\end{array}$ & $\begin{array}{c}\text { CERAD score } \\
\text { (number of cases) }\end{array}$ & $\begin{array}{c}\text { Braak stage } \\
\text { (number of cases) }\end{array}$ & $\begin{array}{c}\text { FBS } \\
(\mathrm{mg} / \mathrm{d} l)\end{array}$ \\
\hline $\mathrm{AD}$ & $19(6 / 13)$ & $85.3 \pm 6.4$ & $7.3 \pm 3.7$ & $\mathrm{~B}(3), \mathrm{C}(16)$ & $\begin{array}{l}\text { III (5), IV (3), } \\
\text { V (4), VI (7) }\end{array}$ & $133 \pm 67$ \\
\hline Non-AD & $21(8 / 13)$ & $83.5 \pm 8.0$ & & $\mathrm{~A}$ & I & $123 \pm 46$ \\
\hline
\end{tabular}

AD: Alzheimer's disease, CERAD: Consortium of Establish a Registry for Alzheimer's Disease, FBS: fasting blood glucose 

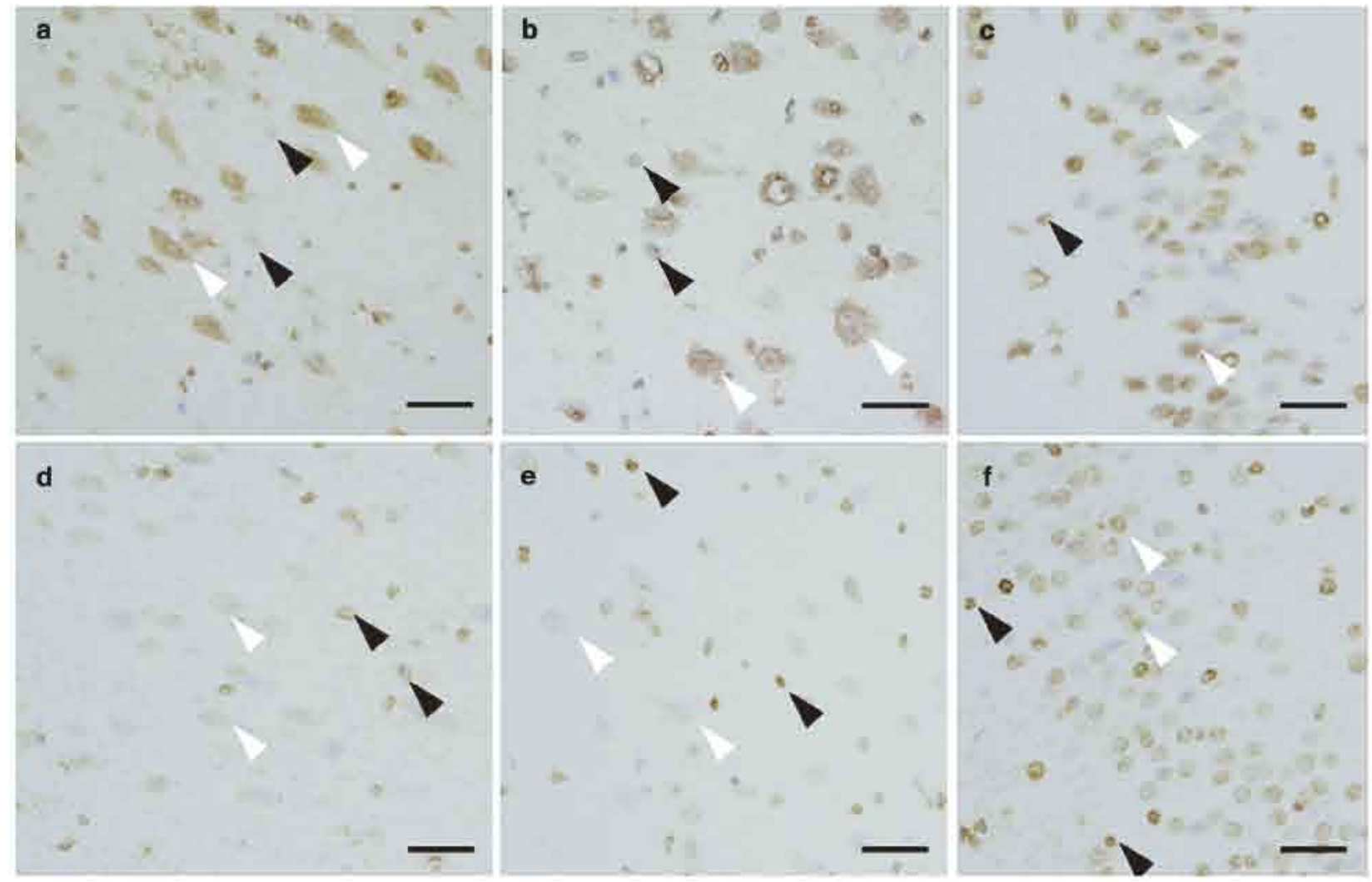

Fig. 1. Immunohistochemistry for endogenous secretory receptor for advanced glycation endproducts (esRAGE) in the hippocampus in non-Alzheimer's disease (non-AD) (a-c) and Alzheimer's disease (AD) (d-f). In AD, CA1 (d) and CA3 (e) pyramidal cells demonstrated low esRAGE immunoreactivities compared with CA1 (a) and CA3 (b) pyramidal cells in non$\mathrm{AD}$; however, the immunoreactivities of dentate granule cells in AD (f) appeared comparable with those in non-AD (c). In CA1 (d) and CA3 (e), the esRAGE immunoreactivities in astrocytes appeared to be preserved or increased in AD. Typical pyramidal cells $(\mathbf{a}, \mathbf{b}, \mathbf{d}$, and $\mathbf{e})$ and granule cells (c and $\mathbf{f})$ are indicated by white arrowheads, and astrocytes are indicated by black arrowheads. Scale bars: $20 \mu \mathrm{m}$

collected for Western blot analysis. After determination of protein concentrations by $\mathrm{BCA}$ protein assay kit (Pierce, Rockford, IL, USA) using BSA as a standard, dithiothreitol and bromphenol blue were added to the final concentration of $100 \mathrm{mM}$ and $0.001 \%(\mathrm{w} / \mathrm{v})$, respectively. Fifty microgram of protein were resolved by SDS-PAGE, and then transferred onto an Immobilon-FL membrane (Millipore, Bedford, MA, USA). The membrane was incubated with $0.5 \mu \mathrm{g} / \mathrm{m} l$ esRAGE-specific polyclonal antibody in Can Get Signal solution 1 (Toyobo, Osaka) and then incubated with 1:10,000 diluted infrared fluorescent dye-labeled anti-rabbit IgG antibody (LI-COR Bioscience, Lincoln, NE, USA) in Can Get Signal solution 2 (Toyobo). The immunoreactive bands were visualized and quantified by Odyssey infrared fluorescence imaging system (LI-COR Bioscience).

\section{Statistic analysis}

Differences among groups were tested using parametric tests [if normally distributed, Student's t test or ANOVA combined with a multiple comparison test (Scheffe's type)] or non-parametric test [if not normally distributed, a Mann-Whitney U test]. $P$ values under $5 \%$ were considered to indicate statistical significance. All analyses were performed with the use of StatView software (SAS institute). 

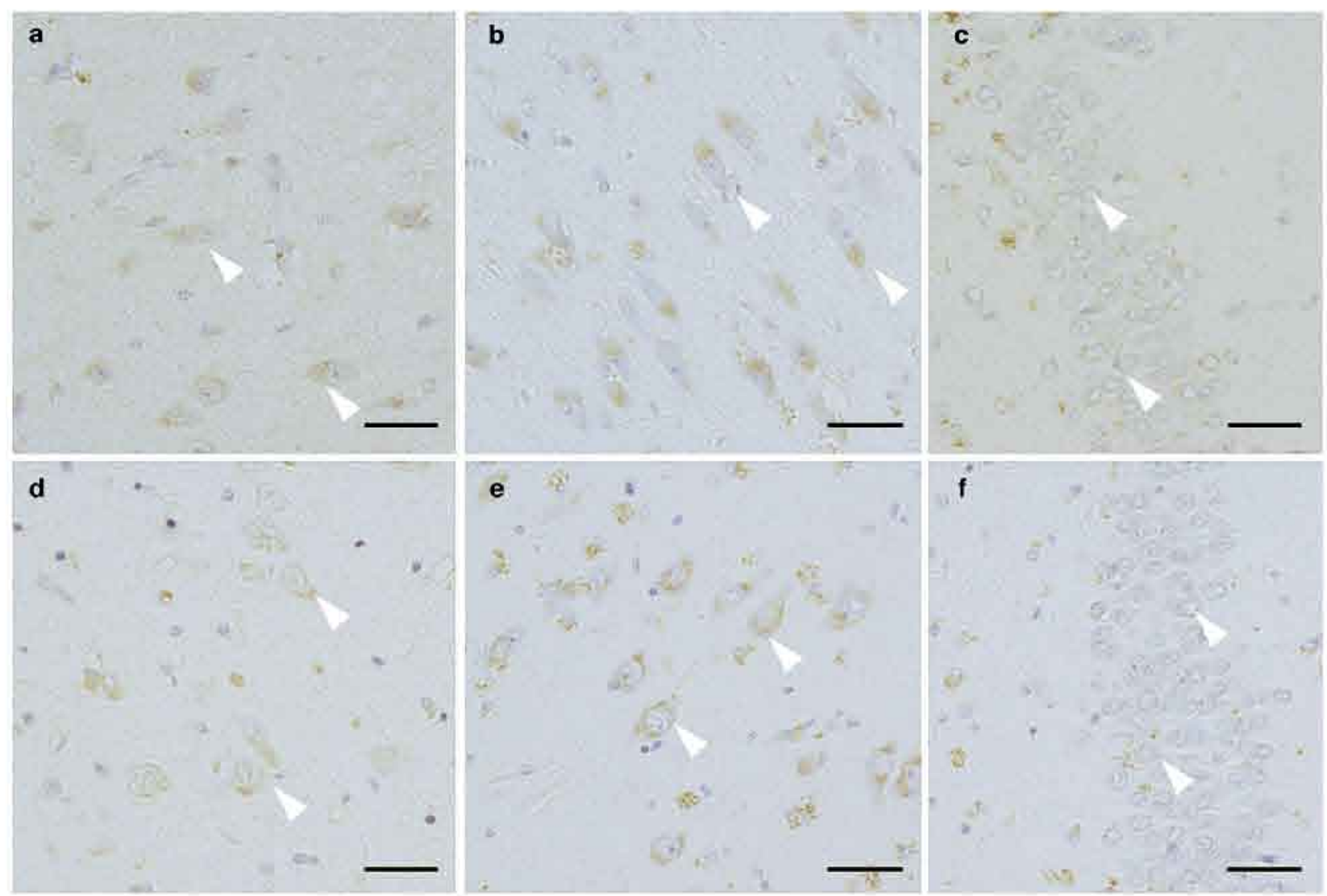

Fig. 2. Immunohistochemistry for membrane-type RAGE in the hippocampus in non-AD (a-c) and in AD (d-f). The expression of membrane-type RAGE in neurons and astrocytes in CA1 (a and d) and CA3 (b and e) was observed. In AD, CA1 (d) and CA3 (e) pyramidal cells demonstrated almost the same membrane-type RAGE immunoreactivities as CA1 (a) and CA3 (b) pyramidal cells in non-AD, respectively. The immunoreactivities in dentate granule cells were weak both in AD (f) and non-AD (c). Typical pyramidal cells (a, b, d, and $\mathbf{e})$ and granule cells (c and f) are indicated by white arrowheads. Scale bars: $20 \mu \mathrm{m}$

\section{Results}

\section{Cases}

Table 1 summarizes age, sex, clinical duration, neuropathological findings and other information. There were no significant differences in age and the fasting blood glucose level between the AD and non-AD group. In the AD group, the CERAD score was $B(n=3)$ or $C(n=16)$, and the Braak stage was III $(n=5)$, IV $(n=3), V(n=4)$, or VI $(n=7)$. In the non-AD group, the CERAD score was all $\mathrm{A}$, and the Braak stage was all $\mathrm{I}$.

\section{Neuropathology and immunohistochemistry}

In the hippocampus of $\mathrm{AD}$, loss of the pyramidal cells in CA1 region was apparent in contrast to preservation of the pyramidal cells in CA3 region and the granule cells in dentate gyrus as reported previously (West et al., 2004).

In the immunostaining for esRAGE, diffuse or dotlike cytoplasmic staining was observed in pyramidal cells, coarse cytoplasmic staining was observed in dentate granule cells, and diffuse cytoplasmic staining was observed in astrocytes. The immunoreactivities of esRAGE were varied in different regions of the hippocampal formation. In the non-AD group, strong immunoreactivities of esRAGE were observed in the pyramidal cells at $\mathrm{CA} 1$ and $\mathrm{CA} 3$, and in the 


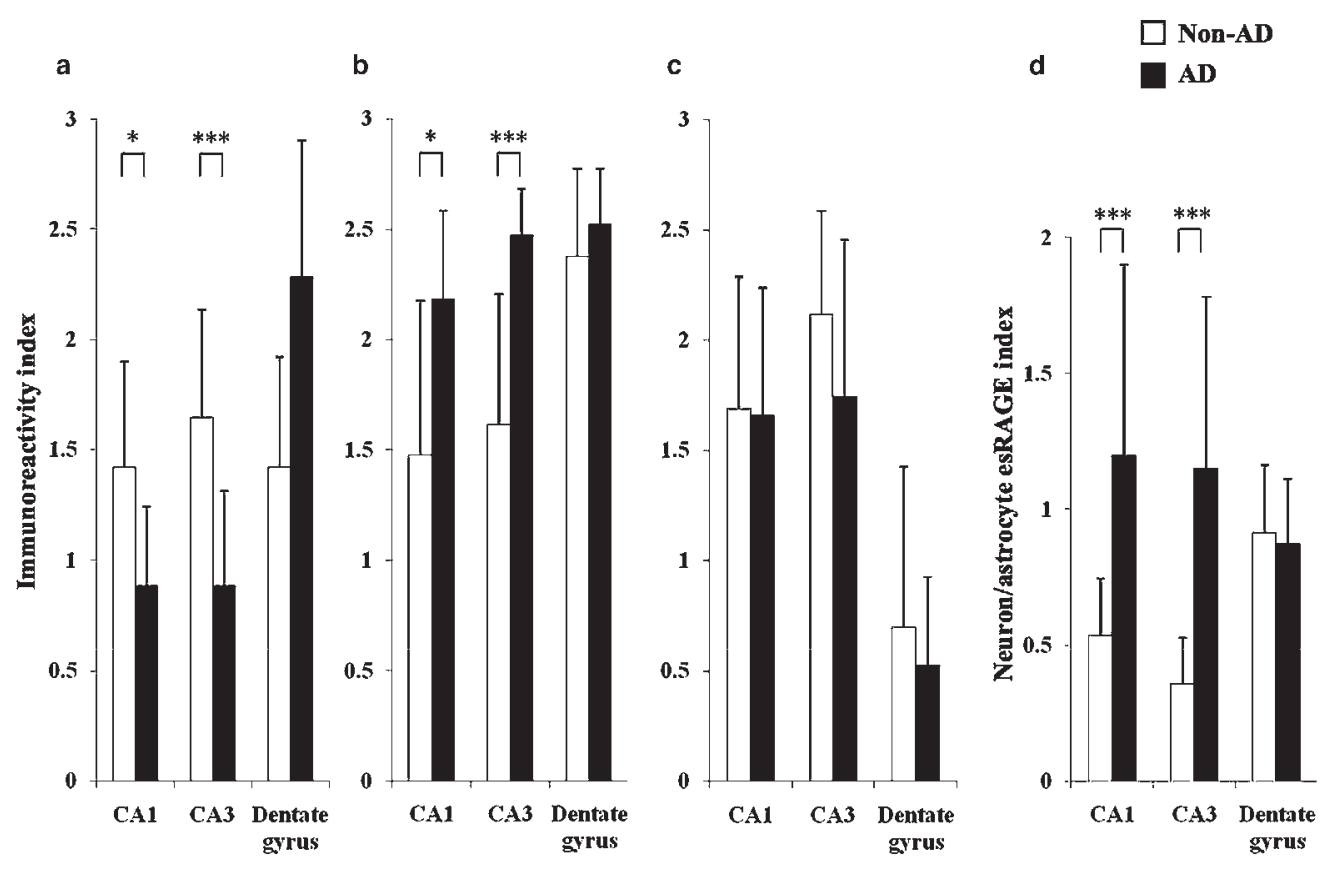

Fig. 3. Semiquantitative evaluation of immunoreactivity for esRAGE (a, b) and membrane-type RAGE (c) in each hippocampal subdivision. Neurons $(\mathbf{a}, \mathbf{c})$ and astrocytes $(\mathbf{b})$ were separately evaluated, and neuron/ astrocytes esRAGE index was calculated as described in the text (d). a: The esRAGE-immunoreactivity indexes (IRI) in pyramidal cells significantly decreased in CA1 region $\left({ }^{*} p<0.05\right)$ and CA3 region $\left({ }^{* * *} p<\right.$ 0.001 ) of $\mathrm{AD}$ compared with non- $\mathrm{AD}$, and there was no significant statistical difference in dentate granule cells between AD and non-AD. b: esRAGE IRI in astrocytes were significantly increased in CA1 region $\left(^{*} p<\right.$ $0.05)$ and in $\mathrm{CA} 3$ region $\left({ }^{* *} p<0.001\right)$ of $\mathrm{AD}$ compared with non- $\mathrm{AD}$, and there was no significant statistical difference in dentate gyri between $\mathrm{AD}$ and non-AD. $\mathbf{c}$ : The IRI of membrane-type RAGE in pyramidal cells in CA1 and CA3 regions, and in dentate granular cells were not significantly different between AD and nonAD. d: The neuron/astrocyte esRAGE indexes significantly decreased in CA1 region $\left({ }^{* * *} p<0.001\right)$ and in CA3 region $\left.{ }^{* * *} p<0.001\right)$ of $\mathrm{AD}$ compared with non- $\mathrm{AD}$, and showed no difference in dentate granule cells between $\mathrm{AD}$ and non-AD. Open bars and closed bars indicate means of the immunoreactivity index of non$\mathrm{AD}$ group and $\mathrm{AD}$ group, respectively. Error bars indicate standard deviation.

dentate granule cells. In the AD group, the esRAGE immunoreactivities decreased in surviving CA1 pyramidal cells and in CA3 pyramidal cells, but those in dentate granule cells appeared comparable with the non-AD group. In CA1 and CA3 regions, the esRAGE immunoreactivities in astrocytes appeared to be preserved or increased contrary to the decreased immunoreactivities in pyramidal cells in AD (Fig. 1).

In the immunostaining for membrane-type RAGE, diffuse or coarse cytoplasmic staining was observed in pyramidal cells, dentate granule cells and astrocytes; immunoreactivities of dentate granule cells were weak. Membrane-type RAGE immunoreactivities in the CA1 and CA3 pyramidal cells and dentate granule cells were not apparently different between the $\mathrm{AD}$ and the non-AD group (Fig. 2).

The neuronal IRI of esRAGE significantly decreased at $\mathrm{CA} 1$ region (Fig. 3a; $p<0.05$ ) and $\mathrm{CA} 3$ region of $\mathrm{AD}$ (Fig. $3 \mathrm{a} ; p<0.001$ ), and there was no significant statistical difference in dentate gyri between AD and non-AD (Fig. 3a). However, in the astrocytes, those were significantly increased at CA1 region (Fig. 3b; $p<0.05$ ) and CA3 region of $\mathrm{AD}$ (Fig. $3 \mathrm{~b} ; p<0.001$ ), and showed no difference in dentate gyri between AD and non-AD (Fig. $3 b$ ). In the AD group, a significant decrease of esRAGE immunoreactivity in pyramidal cells was accompanied 
a

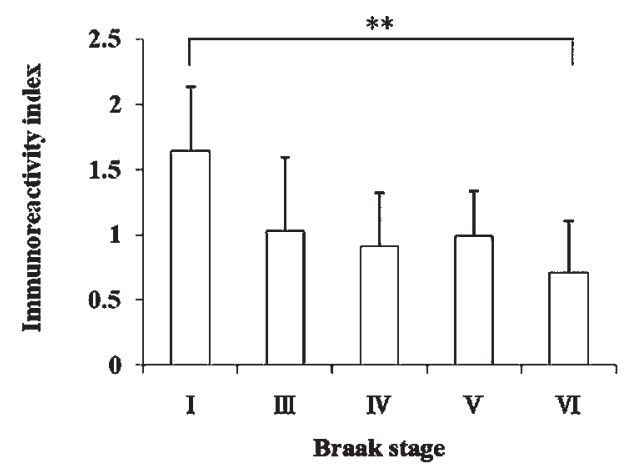

c

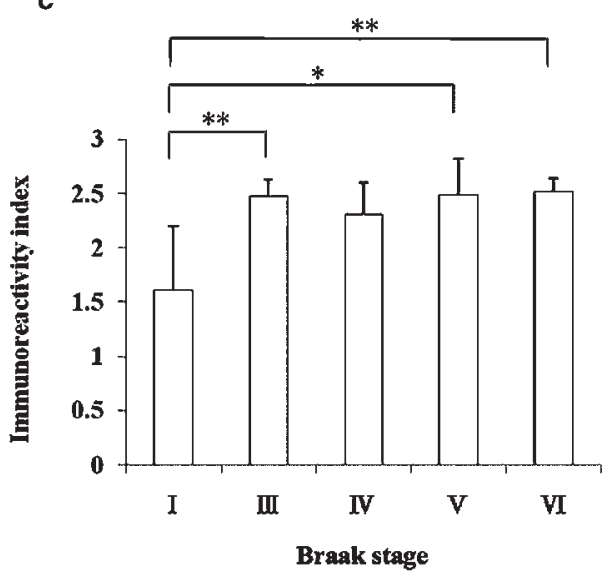

b

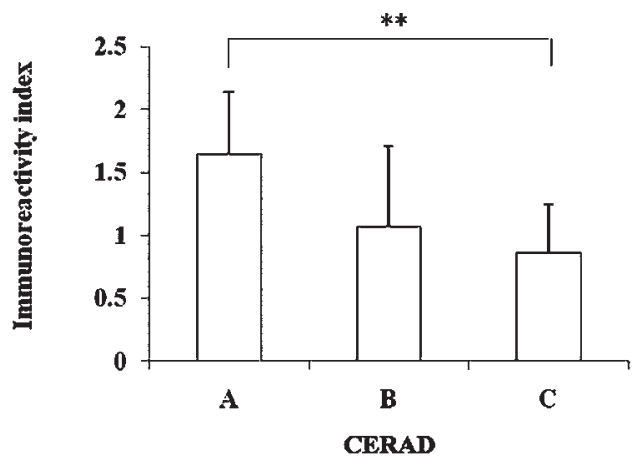

d

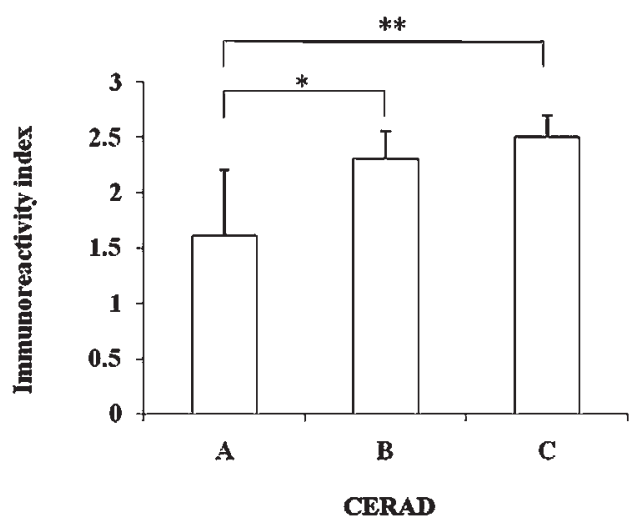

Fig. 4. Semiquantitative evaluation of immunoreactivity for esRAGE in CA3 region classified by pathological staging, Braak staging $(\mathbf{a}, \mathbf{c})$ and CERAD criteria $(\mathbf{b}, \mathbf{d})$. The cases of Stage $\mathrm{I}$ in Braak staging and stage A in CERAD criteria correspond to non-AD specimens. Tendencies of the decrease of immunoreactivity in the pyramidal cells $(\mathbf{a}, \mathbf{b})$ and the increase in astrocytes $(\mathbf{c}, \mathbf{d})$ were observed from the early stages in AD. Error bars indicate standard deviation. ${ }^{*} p<0.05,{ }^{* *} p<0.01$

by a significant increase of the immunoreactivity in astrocytes compared with non-AD group. In contrast to esRAGE, the IRI of membrane-type RAGE in neurons were not significantly different at CA1, CA3 and dentate gyri between AD and non-AD (Fig. 3c). For esRAGE, to evaluate the decreased immunoreactivity in neurons and increased immunoreactivity in astrocytes simultaneously, the neuron/astrocyte esRAGE index (esRAGE IRI for neuron/esRAGE IRI for astrocyte) was calculated. As in the case of esRAGE IRI for neuron, the neuron/astrocyte esRAGE indexes significantly decreased at CA1 region ( $p$ $<0.001)$ and CA3 region of $\mathrm{AD}(p<0.001)$, and showed no difference in dentate gyri between $A D$ and non-AD
(Fig. 3d). When the immunoreactivity of esRAGE in CA3 region was classified according to the pathological staging, CERAD criteria based on semiquantification of senile plaques and Braak staging based on distribution of neurofibrillary tangles, the tendencies of the decrease of esRAGE in neurons and its increase in astrocytes were observed from the early stages in both CERAD and Braak staging (Fig. 4, 5). The tendencies of esRAGE were equivocal in CA1 region (data not shown). No such apparent tendencies were observed for membrane-type RAGE immunoreactivity (data not shown). 


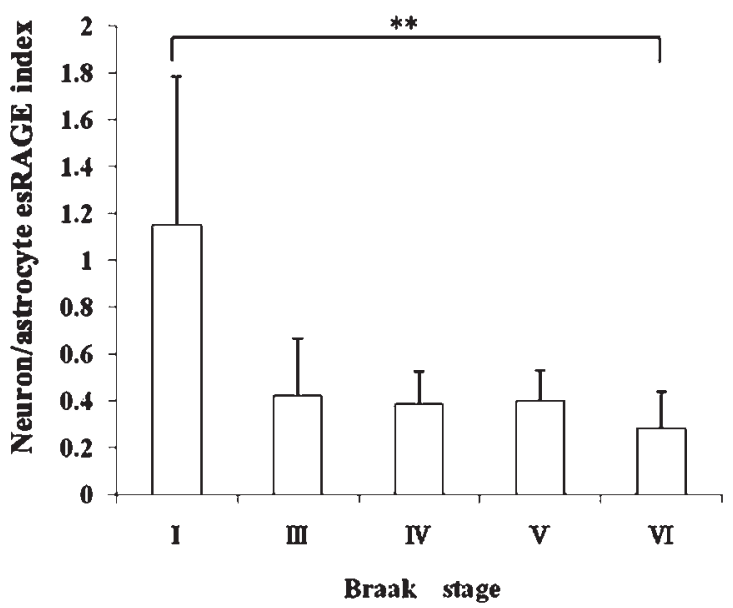

b

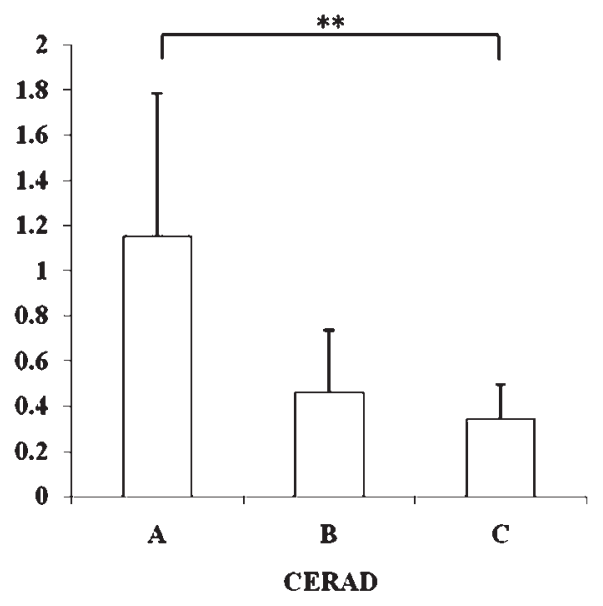

Fig. 5. Neuron/astrocytes esRAGE index classified by histological staging of AD. The neuron/astrocytes esRAGE index was classified by Braak staging (a) or CERAD criteria (b). The index showed a tendency to decrease as pathological changes progress. Error bars indicate standard deviation. ${ }^{* *} p<0.01$

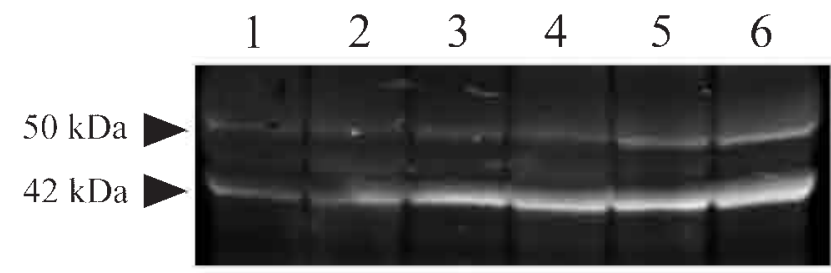

Fig. 6. Western blot analysis of hippocampus homogenates using esRAGE-specific antibody. Fifty microgram protein was loaded on each lane. Detection of immunoreactive bands was carried out using Odyssey infrared fluorescence imaging system. Three independent AD samples (lanes 1-3) and three independent normal aging samples (lanes 4-6) were analyzed. Two major immunoreactive bands at approx. 50 $\mathrm{kDa}$ and at approx. $42 \mathrm{kDa}$ (arrowheads) were detected, and the intensities of the bands in AD samples were lower than those of normal aging samples.

\section{Western blot analysis}

In hippocampus homogenates, two major immunoreactive bands were identified at approx. $50 \mathrm{kDa}$ and at approx. $42 \mathrm{kDa}$ in Western blot analysis using the esRAGE-specific antibody. Three AD samples, whose esRAGE immunoreactivities in pyramidal cells were weak, and three normal aging samples, whose
esRAGE immunoreactivities in pyramidal cells were strong, were analyzed, and the intensities of esRAGE bands in $\mathrm{AD}$ samples were relatively lower $(\sim 35 \%$ decrease on an average) compared with normal aging samples (Fig. 6). This result indicated that the immunoreactivity of esRAGE in neurons in immunostaining correlated with the total amount of esRAGE in the brain tissue.

\section{Discussion}

esRAGE has a protective effect as a decoy receptor, while membrane-bound, full-length RAGE transmits cytotoxic signals (Yonekura et al., 2005). esRAGE is a different entity from sRAGE (soluble RAGE) that includes proteinase-cleaved form of membranetype RAGE or esRAGE (Schmidt et al., 1994; Hanford et $a l ., 2004$ ) and genetically engineered (C-terminal truncated) recombinant soluble RAGE protein (Renard et al., 1997; Park et al., 1998); esRAGE is unique in that it is endogenously generated secretory isoform of RAGE. The esRAGE-specific antibody used in this study recognizes the unique C-terminal region of esRAGE, and does not crossreact with proteinase-cleaved form of RAGE. We have recently reported the expression of esRAGE in neurons in human and mouse brain (Cheng 
et al., 2005; Harashima et al., 2006). The administration of purified SRAGE was reported to inhibit membranetype RAGE-mediated transport of $\mathrm{A} \beta$ across blood-brain barrier and to suppress $A \beta$ accumulation in mouse brain parenchyma (Dean et al., 2003). In AD brains, esRAGE would capture $A \beta$ and reduce cytotoxicity induced by $\mathrm{A} \beta$, and the levels of esRAGE expression may be associated with the vulnerability of neurons. AGE that has been reported to localize in the neurons in the hippocampus and parahippocampal gyrus in AD brain may also be RAGE ligand responsible for AD pathogenesis (Sato et al., 2006).

The immunohistochemistry for esRAGE in the present study demonstrated that esRAGE-positive neurons and astrocytes were detectable in hippocampus in both $\mathrm{AD}$ and non-AD brains (Fig. 1, 2). Semiquantitative evaluation of immunoreactivity of neurons in AD hippocampus showed a significant decrease of esRAGE immunoreactivities in the pyramidal cells in CA1 and CA3 regions compared with non- $A D$ although the dentate granule cells showed no difference of esRAGE immunoreactivity between $A D$ and non-AD (Fig. 3). In $\mathrm{AD}$, there is selective neuronal loss that is critical for cognitive function. The pyramidal cells in the CA1 region of hippocampus are vulnerable to degeneration, whereas the CA3 pyramidal cells and dentate granule cells are resistant to degeneration (West et al., 2004). The decrease of esRAGE immunoreactivity of CA3 pyramidal cells, which are relatively preserved in $A D$, indicates that the decrease of esRAGE immunoreactivity in neurons is not simply a reflection of neuronal cell death. The selective loss of CA1 pyramidal cells may be a consequence of reduced protection by esRAGE and CA1-specific neuronal vulnerability.

Immunohistochemistry in $\mathrm{AD}$ brains demonstrated an increase of esRAGE immunoreactivity in astrocytes compared with non-AD contrary to the decrease of immunoreactivity in pyramidal cells. The induction of the esRAGE expression in astrocytes may reflect the inflammatory process in AD brains (Akiyama et al., 2000).

In CA3 region, the above mentioned tendencies, that is, the decrease of esRAGE in the pyramidal cells and it's increase in the astrocytes, were apparent in the early stages of pathological classifications of AD (i.e. CERAD criteria and Braak staging) (Fig. 4). It is noteworthy that the tendencies were apparent in the limbic stages (stages III and IV) of Braak staging, the stages that do not directly correlate with clinical dementia. Those tendencies were equivocal in CA1 region, which may be due to modification by neuronal loss at later stages in CA1 region (data not shown). No apparent tendencies were observed for membrane-type RAGE immunoreactivity (data not shown).

To evaluate decreased immunoreactivity in neurons and increased immunoreactivity in astrocytes simultaneously, the neuron/astrocyte esRAGE index (esRAGE IRI for neuron/esRAGE IRI for astrocyte) was calculated. Since the statistical significance of the difference of the neuron/ astrocyte esRAGE index between the AD and non-AD group was more than esRAGE IRI for neurons or esRAGE IRI for astrocytes (Fig. 3), the decrease of the neuron/ astrocyte esRAGE index may be more sensitive and useful than simply using these IRI as a diagnostic index for $\mathrm{AD}$. The decrease of neuron/astrocyte esRAGE index was observed from the early pathological stages classified by CERAD or Braak staging (Fig. 5). Although the neuron/astrocyte esRAGE index did not show statistically significant differences in the early stages of pathological changes, in which the esRAGE IRI for astrocyte showed statistically significant differences (Fig. 4c, d), the neuron/ astrocyte esRAGE index would be more specific for $A D$ than esRAGE IRI for astrocytes that could increase in any inflammatory disease.

Western blot analysis of hippocampus homogenates using the esRAGE-specific antibody revealed two major immunoreactive bands at approx. $50 \mathrm{kDa}$ and at approx. $42 \mathrm{kDa}$. In our previous Western blot analysis on lysate of COS-7 cells transfected with human-esRAGE cDNAexpression vector, $\mathrm{N}$-glycosylated $50-\mathrm{kDa}$ esRAGE and unmodified 46-kDa esRAGE were identified (Yonekura et al., 2003). The 50-kDa immunoreactive band in hippocampus homogenates corresponds to the N-glycosylated form of esRAGE. The 42-kDa immunoreactive band might be a partially proteolytic form of esRAGE. The intensities of the immunoreactive bands in the homogenates of AD hippocampus were relatively lower than those of the normal aging samples, and this might reflect the decrease of esRAGE expression in neurons and/or a decrease in the number of neurons.

Since the neuronal expression levels of membrane-type RAGE in AD were almost same as in non-AD (Fig. 2), the selective reduction of neuronal esRAGE expression may be involved in AD. The balance of production between esRAGE and membrane-type RAGE is regulated by alternative 5 -splice site selection of intron 9 in human RAGE mRNA (Yonekura et al., 2003), and some nuclear factors appeared to be involved in the regulatory mechanism (K.O., T.W., and H.Y., unpublished data). In the process of AD pathology, the expression or functions of these factors may be altered.

Donahue et al. reported the decrease of immunoreactivity of RAGE in hippocampal neurons of AD using immunohistochemical methods (Donahue et al., 2006). Since the antibody used in their study was raised 
against the extracellular domain of RAGE and was supposed to recognize both membrane-type RAGE and esRAGE, their results were consistent with the decreased esRAGE and the unchanged membrane-type RAGE immunoreactivity in our results. However, the Western blot analysis in their study indicated an increase of total RAGE protein in hippocampal tissue of AD. Because different antibodies were used and because of the small number of samples (three AD and three controls in our study; one AD and one control in their study), there is room for further investigation using isoform-specific antibodies for more samples.

esRAGE detected in neurons appeared to be intracellular storage of esRAGE and/or esRAGE on the secretory pathway. Although the immunoreactivity of esRAGE in extracellular space was not apparent in this study, the amount of secreted esRAGE is supposed to correspond to its expression level, which is indicated by esRAGE immunoreactivity in the cell. Since it was suggested that sRAGE and esRAGE bind heparan sulfate on the cell membranes or in the extracellular matrix (Hanford et al., 2004), it is probable that the secreted esRAGE does not diffuse out but localizes and captures the ligands on the cell surface and/or in the vicinity of the cell. This may explain the observation that the increase of esRAGE expression in astrocytes did not appear to be effective for protection of neurons.

In conclusion, esRAGE appeared to be involved in $A D$ pathology in the subdivision specific manner in hippocampus. Decreased neuronal expression of esRAGE in CA1 and CA3 regions is correlated with elevated glial expression of esRAGE in AD brains. The onset and/or progression of $\mathrm{AD}$ might be associated with reduction of esRAGE in the pyramidal cells. The neuronal expression of esRAGE should thus be a novel diagnostic marker of $\mathrm{AD}$ and could have a therapeutic potential in the treatment of AD.

\section{Acknowledgments}

We thank, Kenji Kosaka and Yoshio Hashizume for neuropathological examinations, Yuji Fujinawa and Osamu Yamamoto for their excellent technique and Shinichi Matsudaira and Reiko Kitamura for assistance.

\section{References}

Akatsu H, Takahashi M, Matsukawa N, Ishikawa Y, Kondo N, Sato T, Nakazawa H, Yamada T, Okada H, Yamamoto T, Kosaka K: Subtype analysis of neuropathologically diagnosed patients in a Japanese geriatric hospital. J Neurol Sci 196: 63-69 (2002).

Akiyama H, Barger S, Barnum S, Bradt B, Bauer J, Cole GM, Cooper NR, Eikelenboom P, Emmerling M, Fiebich BL, Finch CE, Frautschy S, Griffin WS, Hampel H, Hull M, Landreth G, Lue L, Mrak R, Mackenzie IR, McGeer PL, O'Banion MK, Pachter J, Pasinetti G, Plata-Salaman C, Rogers J, Rydel R, Shen Y, Streit W, Strohmeyer R, Tooyoma I, Van Muiswinkel FL, Veerhuis R, Walker D, Webster S, Wegrzyniak B, Wenk G, Wyss-Coray T: Inflammation and Alzheimer's disease. Neurobiol Aging 21: 383-421 (2000).

Braak H, Braak E: Neuropathological stageing of Alzheimer-related changes. Acta Neuropathol 82: 239259 (1991).

Cheng C, Tsuneyama K, Kominami R, Shinohara H, Sakurai S, Yonekura H, Watanabe T, Takano Y, Yamamoto H, Yamamoto Y: Expression profiling of endogenous secretory receptor for advanced glycation end products in human organs. Mod Pathol 18: 13851396 (2005).

Deane R, Du Yan S, Submamaryan RK, LaRue B, Jovanovic S, Hogg E, Welch D, Manness L, Lin C, Yu J, Zhu H, Ghiso J, Frangione B, Stern A, Schmidt AM, Armstrong DL, Arnold B, Liliensiek B, Nawroth P, Hofman F, Kindy M, Stern D, Zlokovic B: RAGE mediates amyloid-beta peptide transport across the blood-brain barrier and accumulation in brain. Nat Med 9: 907-913 (2003).

Donahue JE, Flaherty SL, Johanson CE, Duncan JA 3rd, Silverberg GD, Miller MC, Tavares R, Yang W, Wu Q, Sabo E, Hovanesian V, Stopa EG: RAGE, LRP-1, and amyloid-beta protein in Alzheimer's disease. Acta Neuropathol 112: 405-415 (2006).

Hanford LE, Enghild JJ, Valnickova Z, Petersen SV, Schaefer LM, Schaefer TM, Reinhart TA, Oury TD: Purification and characterization of mouse soluble receptor for advanced glycation end products (sRAGE). J Biol Chem 279: 50019-50024 (2004).

Harashima A, Yamamoto Y, Cheng C, Tsuneyama K, Myint KM, Takeuchi A, Yoshimura K, Li H, Watanabe T, Takasawa S, Okamoto H, Yonekura H, Yamamoto $\mathrm{H}$ : Identification of mouse orthologue of endogenous secretory receptor for advanced glycation end-products: structure, function and expression. Biochem J 396: 109- 
115 (2006).

Hofmann MA, Drury S, Fu C, Qu W, Taguchi A, Lu Y, Avila C, Kambham N, Bierhaus A, Nawroth P, Neurath MF, Slattery T, Beach D, McClary J, Nagashima M, Morser J, Stern D, Schmidt AM: RAGE mediates a novel proinflammatory axis: a central cell surface receptor for $\mathbf{S 1 0 0 / c a l g r a n u l i n ~ p o l y p e p t i d e s . ~ C e l l ~ 9 7 : ~}$ 889-901 (1999).

Hori O, Brett J, Slattery T, Cao R, Zhang J, Chen JX, Nagashima M, Lundh ER, Vijay S, Nitecki D, Morser J, Stern D, Schmidt AM: The receptor for advanced glycation end products (RAGE) is a cellular binding site for amphoterin. $J$ Biol Chem 270: 25752-25761 (1995).

Huttunen HJ, Fages C, Rauvala H: Receptor for advanced glycation end products (RAGE)-mediated neurite outgrowth and activation of NF- $\kappa$ B require the cytoplasmic domain of the receptor but different downstream signaling pathways. J Biol Chem 274: 19919-19924 (1999).

Koyama H, Shoji T, Yokoyama H, Motoyama K, Mori K, Fukumoto S, Emoto M, Shoji T, Tamei H, Matsuki H, Sakurai S, Yamamoto Y, Yonekura H, Watanabe T, Yamamoto H, Nishizawa Y: Plasma level of endogenous secretory RAGE is associated with components of the metabolic syndrome and atherosclerosis. Arterioscler Thromb Vasc Biol 25: 2587-2593 (2005).

Kumada T, Tsuneyama K, Hatta H, Ishizawa S, Takano Y: Improved 1-h rapid immunostaining method using intermittent microwave irradiation: practicability based on 5 years application in Toyama Medical and Pharmaceutical University Hospital. Mod Pathol 17: 1141-1149 (2004).

Lander HM, Tauras JM, Ogiste JS, Hori O, Moss RA, Schmidt AM: Activation of the receptor for advanced glycation end products triggers a 21 (ras)-dependent mitogen-activated protein kinase pathway regulated by oxidant stress. J Biol Chem 272: 17810-17814 (1997).

Lue LF, Walker DG, Rogers J: Modeling microglial activation in Alzheimer's disease with human postmortem microglial cultures. Neurobiol Aging 22: 945-956 (2001).

Mackic JB, Stins M, McComb JG, Calero M, Ghiso J, Kim KS, Yan SD, Stern D, Schmidt AM, Frangione, B, Zlokovic BV: Human blood-brain barrier receptors for Alzheimer's amyloid-beta 1-40. Asymmetrical binding, endocytosis, and transcytosis at the apical side of brain microvascular endothelial cell monolayer. $J$ Clin Invest 102: 734-743 (1998).
Mirra SS, Heyman A, McKeel D, Sumi SM, Crain BJ, Brownlee LM, Vogel FS, Hughes JP, van Belle G, Berg L: The Consortium to Establish a Registry for Alzheimer's Disease (CERAD). Part II. Standardization of the neuropathologic assessment of Alzheimer's disease. Neurology 41: 479-486 (1991).

Park L, Raman KG, Lee KJ, Lu Y, Ferran LJ Jr, Chow WS, Stern D, Schmidt AM: Suppression of accelerated diabetic atherosclerosis by the soluble receptor for advanced glycation endproducts. Nat Med 4: 1025-1031 (1998).

Renard C, Chappey O, Wautier MP, Nagashima M, Lundh E, Morser J, Zhao L, Schmidt AM, Scherrmann JM, Wautier JL: Recombinant advanced glycation end product receptor pharmacokinetics in normal and diabetic rats. Mol Pharmacol 52: 54-62 (1997).

Sakurai S, Yamamoto Y, Tamei H, Matsuki H, Obata KI, Hui L, Miura J, Osawa M, Uchigata Y, Iwamoto Y, Watanabe T, Yonekura H, Yamamoto H: Development of an ELISA for esRAGE and its application to type 1 diabetic patients. Diabetes Res Clin Pract 73: 158-165 (2006).

Sato T, Shimogaito N, Wu X, Kikuchi S, Yamagishi S, Takeuchi M: Toxic advanced glycation end products (TAGE) theory in Alzheimer's disease. Am J Alzheimers Dis Other Demen 21: 197-208 (2006).

Schmidt AM, Vianna M, Gerlach M, Brett J, Ryan J, Kao J, Esposito C, Hegarty H, Hurley W, Clauss M, Wang F, Pan YCE, Tsang TC, Stern D: Isolation and characterization of two binding proteins for advanced glycosylation end products from bovine lung which are present on the endothelial cell surface. J Biol Chem 267: 14987-14997 (1992).

Schmidt AM, Hori O, Brett J, Yan SD, Wautier JL, Stern D: Cellular receptors for advanced glycation end products. Implications for induction of oxidant stress and cellular dysfunction in the pathogenesis of vascular lesions Arterioscler Thromb 14: 1521-1528 (1994).

Sousa MM, Yan SD, Stern D, Saraiva MJ: Interaction of the receptor for advanced glycation end products (RAGE) with transthyretin triggers nuclear transcription factor $\kappa \mathrm{B}(\mathrm{NF}-\kappa \mathrm{B})$ activation. Lab Invest 80 : 1101$1110(2000)$.

Stren DM, Schmidt AM, Yan SD, Yan SF : Receptor for advanced glycation endproducts (RAGE) and the complications of diabetes. Ageing Res Rev 1: 115(2002).

West MJ, Kawas CH, Stewart WF, Rudow GL, Troncoso JC: Hippocampal neurons in pre-clinical Alzheimer's disease. Neurobiol Aging 25: 1205-1212 (2004). 
Yan SD, Chen X, Fu J, Chen M, Zhu H, Roher A, Slattery T, Zhao L, Nagashima M, Morser J, Migheli A, Nawroth P, Stern D, Schmidt AM: RAGE and amyloid$\beta$ peptide neurotoxicity in Alzheimer's disease. Nature 382: 685-691 (1996).

Yonekura H, Yamamoto Y, Sakurai S, Petrova RG, Abedin Md J, Li H, Yasui K, Takeuchi M, Makita Z, Takasawa S, Okamoto H, Watanabe T, Yamamoto $H$ : Novel splice variants of the receptor for advanced glycation end-products expressed in human vascular endothelial cells and pericytes, and their putative roles in diabetes-induced vascular injury. Biochem $J 370$ : 1097-1109 (2003).

Yonekura H, Yamamoto Y, Sakurai S, Watanabe T, Yamamoto $\mathrm{H}$ : Roles of the receptor for advanced glycation endproducts in diabetes-induced vascular injury. J Pharmacol Sci 97: 305-311 (2005). 